\title{
¿Qué esperan encontrar los alumnos en los estudios de Psicología? Metas y motivos personales de los estudiantes en su primer año en la universidad
}

\author{
Elena Gámez*, Hipólito Marrero, Jose M. Díaz y Mabel Urrutia
}

Universidad de La Laguna

\begin{abstract}
Resumen: Numerosas investigaciones se han interesado por el perfil motivacional de ingreso de los universitarios. Sin embargo, se ha prestado menos atención al estudio de las metas que los estudiantes pretenden satisfacer cuando llegan a la universidad. En este trabajo presentamos un primer estudio con 610 alumnos de Psicología, de cuatro cursos consecutivos. En él se analiza la estructura factorial de una escala que evalúa la identificación de los estudiantes con diferentes tipos de motivos. En un segundo estudio, con otros 140 estudiantes, se relacionan las metas interpersonales con otras metas vitales, la satisfacción de necesidades psicológicas, el bienestar subjetivo y la presencia de pensamientos perfeccionistas. Los datos muestran la importancia de las metas de logro, de poder, de afiliación y de superación de problemas personales. Estas últimas presentan, además, una relación negativa con el bienestar y la satisfacción de necesidades psicológicas, y una relación positiva con el perfeccionismo. Nuestros resultados aportan un mayor conocimiento de los perfiles de los estudiantes de nuevo ingreso y las consecuencias que de ellos se derivan para su orientación académica. Palabras clave: Metas interpersonales; motivos estudiantes; necesidades psicológicas; bienestar subjetivo; perfeccionismo.
\end{abstract}

\section{Introducción}

Uno de los últimos informes de la Organización para la Cooperación y el Desarrollo Económico (OECD, 2011) sobre el estado de la educación en 26 países del mundo constata que en la mayoría de ellos se ha incrementado el porcentaje de los estudiantes que se gradúan en educación secundaria específica para ir a la universidad. Actualmente la media en el grupo de países más industrializados es del $53 \%$, y en España alcanza el $45 \%$.

En nuestro país, los estudiantes que ingresan en la universidad se caracterizan por tener entre 18 y 19 años y acceder a los estudios superiores como el tránsito "normal" después de superar la formación secundaria. Sin embargo, en lugares como Australia, los países nórdicos o Estados Unidos, una parte importante de los estudiantes de nuevo ingreso tiene 21 años, y accede con experiencia laboral previa. En el informe de la OCDE se analizan las razones de las diferencias en estos perfiles, y los costes y beneficios que tienen ambas opciones. El presente trabajo toma estos datos como punto de partida para analizar el perfil motivacional (en cuanto a metas) de esos estudiantes españoles que ingresan en la universidad.

Las metas no son sólo creencias, es decir representaciones cognitivas sobre lo que deseamos, sino que están conectadas directamente con el entorno y la acción. Como dicen Moscowitz y Grant (2009, p.10):

* Dirección para correspondencia [Correspondence address]:

Elena Gámez. Facultad de Psicología. Campus de Guajara s/n, 38071, La Laguna, S/C de Tenerife (España).E-mail: egamez@ull.es
Title: What do students expect to find when they enter Psychology studies? Goals and personal motives during the first year at university.

Abstract: This study investigated Psychology students' interpersonal and social goals during their first year at university. In Study 1 we analyze the reliability and ran a confirmatory factor analysis of a 45 -item questionnaire about different kinds of interpersonal motives. The sample was integrated by 610 first year college students from four consecutive courses. In a second study with another 140 students, beside the scale about interpersonal goals they completed a set of scales about their aspirations, psychological basic needs of autonomy, competence and relatedness, subjective wellbeing and perfectionism cognitions. Data from both studies showed that: a) Importance is given mainly to achievement, power, affiliation and overcoming personal problems goals; b) The presence of overcoming personal problems goals hindered adjustment, indicated by positive associations with all the perfectionism dimensions, and by negative association with subjective wellbeing and frustration of psychological needs. Our results could provide new insight about the profiles of psychological students and the consequences resulting from them for their academic guidance.

Key words: Interpersonal goals; student's motives; psychological needs, wellbeing; perfectionism.

Las metas son la liaison entre, de un lado, los deseos y las necesidades de las personas, y, de otro, las acciones que ponen en marcha para satisfacerlos. Las metas no siempre generan una conducta, pero la mayoría de las conductas están dirigidas por alguna meta.

El estudio que aquí presentamos se encuadra en uno de los ámbitos de investigación sobre las metas, el de su contenido. Un ámbito que incluye actualmente dos aproximaciones empíricas. Por un lado, identificar tipos concretos de metas y sus consecuencias; aquí situaríamos los trabajos sobre los proyectos personales (Nurmi, 1992), las metas de logro (Elliot, 1999) o las metas vitales desde la perspectiva de la Teoría de la Autodeterminación (TAD) (Kasser y Ryan, 1996). En el otro grupo estarían los estudios transculturales, cuyo objetivo es aislar las metas o conjuntos de metas que compartimos todos los seres humanos.

En el contexto académico, dos líneas de investigación que han resultado especialmente fructíferas desde la perspectiva del contenido de las metas son, por un lado, los trabajos sobre las metas de logro, en lo que se ha dado en llamar los modelos de orientación a metas (Covington, 2000) y, por otro lado, el estudio de las diferencias individuales de las metas vitales desde la TAD (Deci y Ryan, 2000; Grouzet et al., 2005).

En el caso de las metas de logro, existe un abanico de investigaciones en todos los niveles académicos (Elliot $\mathrm{y}$ McGregor, 2001; Linnenbrink y Pintrich, 2002), que han dado lugar a propuestas específicas de intervención educativa (Midgley, 2002). En el ámbito de la educación universitaria española, se han encontrado evidencias de combinaciones de múltiples metas asociadas a resultados exitosos en la vida académica (Rodríguez et al., 2001; Valle et al., 2003, 2009). 
En particular, el alumnado que persigue metas para aprender, metas para conseguir mejores resultados que los demás y metas para evitar dar una mala imagen, es el que informa de un mejor rendimiento académico y el que cree tener un nivel más alto de conocimientos.

Los estudiosos de las orientaciones de logro nos han enseñado por qué los estudiantes hacen muchas de sus tareas académicas (por ejemplo, conseguir conocimiento frente a conseguir reconocimiento académico). Esta búsqueda de metas de aprendizaje frente a rendimiento genera un perfil personal que puede incluir metas diferenciadas de cada uno de los estilos (Pintrich, 2000; Elliot y Moller, 2003) o metas múltiples que coexisten sin dificultad (Valle et al., 2009). Para estos autores lo que hay que hacer es potenciar metas de aprendizaje o metas de rendimiento aproximativo, que son las que se relacionan positivamente con los resultados académicos. Es decir, se debería potenciar en los alumnos sacar buenas notas (meta aproximativa de rendimiento) en lugar del evitar sacar malas notas (meta evitativa de rendimiento).

Los teóricos de la TAD (Deci y Ryan, 2000; Reeve, Nix y Hamm, 2003), por su parte, se han interesado especialmente por la entramada relación entre el contexto (facilitador vs. obstaculizador), las necesidades psicológicas que tienen que satisfacer los alumnos (autonomía, competencia y vinculación) y los estilos de autorregulación que se configuran en el desarrollo evolutivo (extrínseco, introyectado, identificado e intrínseco). El contenido de las metas y esos perfiles individuales tienen un papel determinante en la persistencia y continuidad en los estudios (Niemic y Ryan, 2009; Ryan y Deci, 2009; Reeve, 2009).

Desde la TAD, y en relación con el contenido de las metas, la distinción más fructífera es entre metas intrínsecas y extrínsecas. Entre las primeras estarían la búsqueda de relaciones interpersonales, la participación en una comunidad o el crecimiento personal. Entre las metas extrínsecas, el dinero, la fama o la búsqueda de una imagen popular (Kasser y Ryan, 1996). La elección de unas metas u otras es el resultado de un proceso donde intervienen distintos factores: la satisfacción de las necesidades psicológicas de autonomía, competencia y vinculación; el contexto (facilitador u obstaculizador para la satisfacción de las necesidades), y la historia de cada individuo. Todo ello tendrá consecuencias en el rendimiento (Vansteenkiste, Lens y Deci, 2006). Si un profesor/a quiere que sus estudiantes adopten metas más intrínsecas, tendría que facilitar las condiciones necesarias para que puedan satisfacer sus necesidades psicológicas. Por ejemplo, tendría que explicitar los objetivos de lo que hay que hacer y por qué; permitir elegir entre diferentes alternativas; mejorar las relaciones entre los estudiantes, etc. Hace falta algo más que potenciar el rendimiento eficiente, sacar buenas notas o mejorar en las tareas. Es decir, no es suficiente con centrarse en la consecución de las tareas de logro.

Un intento reciente de integrar la dimensión social en el enfoque de las orientaciones de logro lo representa el trabajo de Shim y Ryan (2012) sobre las metas sociales de logro. En un estudio con estudiantes en su primer año en la universi- dad encontraron que aquellos que tenían metas centradas en mejorar las habilidades y relaciones interpersonales (en comparación con los estudiantes con metas para evitar demostrar incompetencia social) mostraron un mejor ajuste al mundo universitario.

En resumen, parece que hay múltiples indicios sobre la influencia que ejercen las metas sociales en la adaptación, el ajuste y el rendimiento académico. Nuestro interés aquí es contribuir a la perspectiva del contenido de las metas, estudiando el papel de las metas sociales interpersonales en el primer año de los estudios de Psicología y reflexionar sobre las consecuencias que podrían tener en la formación del $\mathrm{cu}$ rrículum universitario. Los datos que presentaremos se organizan en dos estudios. En el primero hemos administrado el cuestionario MOPI (Gámez y Marrero 2000; 2003) a una muestra de 610 estudiantes, de cuatro cursos consecutivos. Con esta muestra analizaremos el patrón de metas sociales que aparece más estable y claro en los estudiantes de psicología. En el Estudio 2 exploraremos, con una muestra distinta, la relación de estas metas con otras variables, buscando un perfil más detallado de estos estudiantes y de sus preocupaciones en el primer año en la universidad.

\section{Estudio 1}

Utilizamos la escala MOPI para explorar la constelación de metas sociales que preocupan a los estudiantes en el primer curso de la universidad. En esta escala se preguntaba a los estudiantes por los motivos para elegir la carrera de psicología. Tenían que valorar 45 ítems, cada uno de los cuales representaba una meta personal, interpersonal o social. Los factores resultantes del $A F E$ explicaban el $37 \%$ de la varianza y mostraron una agrupación de metas en cinco factores: un factor de superación de problemas afectivos de temática interpersonal, un factor de logros asociados al prestigio, un factor de logros asociados a las afiliaciones, un factor de logros asociado al poder y la dominancia, y un último factor relacionado con la motivación intrínseca por el conocimiento.

Nuestro primer objetivo será comprobar si las metas de los estudiantes, en su primer curso de la universidad se mantienen similares a las encontradas en estos estudios previos. Relacionado con este primer objetivo, a una submuestra del conjunto de los participantes les pasamos por segunda vez, el cuestionario MOPI, transcurridos seis meses de la primera prueba. La finalidad era establecer la estabilidad de dichas metas una vez que los estudiantes conocen lo que les ofrece los estudios que han elegido. Por último, un tercer objetivo fue comparar los resultados del cuestionario con una prueba abierta, en la que pedimos a los alumnos que explicitaran seis objetivos o metas que pensaran que podían satisfacer al estudiar Psicología. Se trata de buscar una prueba de validez convergente para la escala, al mismo tiempo que explorábamos la existencia de otros deseos de los estudiantes que hubieran podido quedar fuera de la misma. 


\section{Método}

\section{Participantes}

610 estudiantes de primero de la licenciatura y grado de Psicología de la universidad de La Laguna de cuatro cursos consecutivos: 2006-07 ( $n=126), 2007-2008$ ( $n=148), 2008-09$ $(n=110)$ y $2009-10(n=226)$. Del total de participantes 487 eran mujeres $(79.8 \%)$. La edad media fue de 19.1 años $(D T=$ 3.3). Todos participaron de forma voluntaria para obtener créditos extra en una asignatura.

\section{Instrumentos y Procedimiento}

Escala $M O P I$, para conocer las metas y motivos sociales interpersonales de los estudiantes (Gámez y Marrero 2000; 2003) ampliando la escala de evaluación de 1 a 7 (la versión original era de 5 puntos). Cada uno de los 45 ítems expresaba una afirmación que continuaba la frase "He elegido esta carrera porque....". A lo largo de la escala se presentan temas de logro, como mejora personal o superación de un reto, ítems relacionados con la búsqueda de conocimientos, la búsqueda de relaciones, la superación de problemas personales, la búsqueda de prestigio o influencias, dinero, identificación con modelos, etc.

A una parte de la submuestra del curso 2009-10 $(n=$ 170), un mes después de cumplimentar la escala MOPI y como una nueva tarea sin relación con el cuestionario previo, se les pidió que anotaran en una hoja en blanco seis metas u objetivos que pensaban satisfacer al estudiar psicología. Las metas expresadas por los estudiantes fueron clasificadas por dos jueces en un sistema de 8 categorías que incluía:

- Metas de logro (Ejemplos: ser un buen profesional, mejorar mis conocimientos).

- Metas relacionadas con la superación de problemas personales y afectivos (Ejemplos: conocerme en profundidad para mejorar temas personales, aplicar los conocimientos a mi familia o a mis amigos).

- Metas relacionadas con el poder (Ejemplos: acceder a un trabajo bien remunerado y de prestigio, conseguir un status que tenga influencia social).

- Metas relacionadas con la afiliación (Ejemplos: ayudar a las personas con problemas, conocer gente).

- Motivación por el conocimiento (Ejemplos: conocer la mente, conocer los trastornos mentales).

- Motivación extrínseca (Ejemplos: dinero, conseguir una buena casa).

- Metas relacionadas con la identificación con un modelo (Ejemplos: ser como mis padres o ser como un amigo que estudió esta carrera).

- Metas hedonistas (Ejemplos: sentirme bien, pasármelo bien).
A un grupo de 70 alumnos de esta submuestra se le volvió a pasar la escala $M O P I$ seis meses después de la primera administración.

\section{Resultados}

\section{Análisis Factorial Exploratorio (AFE) del MOPI}

En primera lugar se realizó un análisis factorial exploratorio con el mismo procedimiento utilizado en el trabajo original (Gámez y Marrero, 2000; 2003): método de extracción de componentes principales y rotación varimax. Los valores correspondientes a la medida de adecuación muestral de Kaiser-Meyer-Olkin (KMO) y la prueba de esfericidad de Bartlett fueron excelentes $\chi^{2}{ }_{(990)}=12940.831 ; p<.0001$; $K M O=.930)$. Las comunalidades de los ítems se distribuyeron entre .31 y .70, a excepción de los ítems 3, 7 y 45, cuyos valores eran inferiores a .20 por lo que los eliminamos de los análisis posteriores. En la Tabla 1 aparecen los cuatro factores que emergieron tras la rotación, aplicando el Screen Test de Catell, a partir del gráfico de sedimentación que proporciona el SPSS. El punto de inflexión de la línea que une los valores propios se situó en el cuarto factor, cuyo valor propio antes de la rotación era de 1.5, lo que coincide con el valor aconsejado por el Horn's Pararell Analysis (Watkins, 2006). Para retener un ítem su peso factorial tiene que ser $\geq .50$. Rechazamos los ítems 4 y 10, a pesar de que cumplían este criterio porque también saturaban en algún otro factor con valores cercanos a 40 .

Los cuatro factores seleccionados explicaron el 47\% de la varianza. Al Factor I, que incluye 15 ítems, lo hemos denominado de Logro, e incluye logros orientados al mundo académico (como la búsqueda de conocimientos psicológicos, demostrar tener capacidades o evitar el fracaso y mejorar), así como tres ítems relacionados con las relaciones interpersonales y las motivación de afiliación pero que también podrían entenderse como logros de un futuro psicólogo, ("Deseos de ayudar a los demás" o "Llegar a ser solidario con los otros"). El Factor II es un factor de Poder, los 6 ítems que lo constituyen reflejan metas del tipo ser un/a líder, tener prestigio o una posición dominante. El Factor III, es un factor de Afiliación. Está constituido por 5 ítems, en los que se prima buscar la comunicación y relación con otras personas. Por último, el Factor IV, constituido por 3 ítems, es un factor de Superación de Problemas Personales, problemas afectivos que los estudiantes pretenden resolver al estudiar Psicología. En la Tabla 1 también aparecen los índices de fiabilidad de cada uno de los factores con los ítems seleccionados. 
Tabla 1. Resultado del análisis factorial exploratorio con los valores propios, la varianza explicada por cada factor y las $\alpha$ de Cronbach. Sólo aparecen los pesos factoriales de los ítems que se ajustaron a los criterios de selección.

\begin{tabular}{|c|c|c|c|c|}
\hline Items (metas) & $\begin{array}{c}\text { Factor } \\
\text { I }\end{array}$ & $\begin{array}{c}\text { Factor } \\
\text { II }\end{array}$ & $\begin{array}{c}\text { Factor } \\
\text { III }\end{array}$ & $\begin{array}{c}\text { Factor } \\
\text { IV }\end{array}$ \\
\hline 1 Emprender un camino en la vida & 0.56 & & & \\
\hline 2 Siempre deseos de ayudar a los demás & 0.81 & & & \\
\hline 9 Conocer el ser humano, su mente... & 0.67 & & & \\
\hline 14 Tienes las capacidades y motivaciones & 0.57 & & & \\
\hline 15 Para controlar a los demás hay que conocerlos & 0.72 & & & \\
\hline 20 Sabes escuchar a las personas & 0.64 & & & \\
\hline 21 Llegar a ser independiente & 0.67 & & & \\
\hline 22 Supervisar a otras personas & 0.68 & & & \\
\hline 31 Conocer más las relaciones interpersonales & 0.53 & & & \\
\hline 32 Te evitará sentirte fracasado & 0.55 & & & \\
\hline 33 El esfuerzo será productivo & 0.86 & & & \\
\hline 34 Conocer sobre trastornos, el cerebro.. & 0.67 & & & \\
\hline 36 Disfrutas convenciendo a los demás & 0.57 & & & \\
\hline 41 Valorar las capacidades y mejorar & 0.67 & & & \\
\hline 43 Llegar a ser solidarios con los otros & 0.59 & & & \\
\hline
\end{tabular}

5 Te gusta ser un líder

12 Tener nivel social y económico adecuado

25 Puesto de trabajo con prestigio social $\quad 0.64$

29 Posición social mejor que la actual $\quad 0.74$

$\begin{array}{lr}37 . S u p e r a r & 0.73\end{array}$

39 Acceder a una posición dominante $r .68$

11 Mejor comunicación con otras personas $\quad 0.64 \quad 0.53$

16 Buena ocasión para ser amigos 0.66

17 Has conocido personas que estudiaron esto 0.51

18 Te gusta que la gente pida tu opinión $\quad 0.59$

40 Mantener relaciones con otros/as 0.50

35 Problemas afectivos que puede resolver la $\quad 0.70$

38 Persona con problemas que quieres resolver $\quad 0.71$

44 Resolver algún problema personal 0.70

$\begin{array}{lccc}\text { Porcentaje de varianza explicado }(47,11 \%) & 19.2 & 12.2 & 8.5 \\ \text { Valores propios } & 8.6 & 5.5 & 3.8 \\ \alpha \text { de Cronbach } & .87 & 3.2 & .83\end{array}$

$\alpha$ de Cronbach

Análisis de la prueba abierta: metas expresadas por los estudiantes

En la Tabla 2 aparece el conjunto de metas expresado por la submuestra de 170 participantes. Como puede observarse, a la hora de consignar libremente sus metas, nuestros sujetos muestran preferencias más o menos claras. Las metas relacionadas con la adquisición de conocimiento y las metas afiliativas o vinculatorias resultan claramente destacadas.

Tabla 2. Categorización y frecuencia de las metas señaladas en la prueba abierta.

\begin{tabular}{lcccc}
\hline Tipo de meta & $N$ & $\%$ & $\chi^{2}$ & $p$ \\
\hline Motivación de conocimiento & 225 & 25.48 & 154.09 & .001 \\
Afiliación/Vinculación & 178 & 20.16 & & \\
Superación de problemas & 123 & 13.93 & & \\
Logro & 93 & 10.53 & & \\
Hedonismo & 78 & 8.83 & & \\
Poder & 119 & 13.48 & & \\
Motivación extrínseca: dinero & 67 & 7.59 & & \\
& 883 & 100 & & \\
\hline
\end{tabular}

Luego vendría un conjunto con frecuencias intermedias, formado por tres tipos de metas: las asociadas con la superación de problemas personales, las asociadas con el poder, y las metas de logro. Por último, las metas más relacionadas con el componente hedónico y con la obtención de dinero son las menos citadas.

\section{Análisis Factorial Confirmatorio (AFC) del MOPI}

Con vistas a conocer mejor la constelación de metas se realizó un $A F C$ utilizando el paquete estadístico AMOS 18.0. Antes de llevar a cabo el $A F C$ se analizó la normalidad multivariante de los datos mediante el índice de Mardia, cuyo valor fue más alto de lo deseable (curtosis multivariado = 7.18). En casos como éste, según los especialistas en el $A F C$ (Byrne, 2010), el método de máxima verosimilitud (que es el más utilizado) puede ser problemático. Una alternativa es el método $A D F$, distribución libre asintótica. Para utilizar este método el tamaño de la muestra debe ser, como mínimo 10 veces mayor que el número de parámetros estimados. En 
nuestro caso, el modelo cuenta con 37 parámetros, por lo que el tamaño de la muestra nos permite aplicar este método. Lo más adecuado para aceptar o rechazar un modelo es emplear una combinación de varios índices: $\chi^{2}$ debería tener un valor no significativo $(p>.05)$, pero es muy sensible al tamaño de la muestra (Jöreskog y Sörbom, 1993) por lo que se suele calcular el $\chi^{2} / g l$ que se considera aceptable cuando es $<5$, (Bentler, 1990). Los índices CFI, IFI y GFI se basan en la comparación entre el modelo hipotetizado y el modelo nulo, considerándose aceptables los valores superiores a 0.90. Por último aunque el RMSE $A$ con valores $<.05$ se considera un indicador de un buen modelo, valores cercanos al .06 también indican que el modelo se ajusta bien a los datos (Hu y Bentler, 1999). En la Tabla 3 aparecen los modelos puestos a prueba en el análisis confirmatorio. El primer modelo fue el que resultó del $A F E$. Como se observa en la tabla, su adecuación era insatisfactoria, por lo que pusimos a prueba los modelos 2,3 y 4, donde se eliminaron ítems en todos los factores utilizando la tabla de MI (Modification Index) que nos indica los ítems que tienen un peso en más de un factor o los errores en las covarianzas.
Tabla 3. Índices de bondad de ajuste de cuatro posibles modelos del análisis confirmatorio del MOPI

\begin{tabular}{lllllll}
\hline Modelo & $\chi^{2}$ & $d f$ & CFI & IFI & GFI & RMSEA \\
\hline Modelo 1 & 3065.348 & 371 & .52 & .50 & .83 & .104 \\
Modelo 2 & 1037.273 & 203 & .57 & .52 & .87 & .083 \\
Modelo 3 & 520.886 & 113 & .67 & .60 & .91 & .078 \\
Modelo 4 & 147.701 & 54 & .90 & .91 & .97 & .053 \\
\hline
\end{tabular}

El modelo 4 (representado en la Figura 1) fue el que consiguió los mejores niveles de ajuste. Se han eliminado 11 ítems del factor de logro, 3 ítems del factor de poder, el ítem 18 del factor de afiliación y el ítem 38 del factor de superación de problemas. También se incorpora la covarianza entre los errores de los ítems 11 y 17. El ítem 16 ("Es una buena ocasión para hacer amigos") apareció relacionado con el factor de logro, y el ítem 29 ("Conseguir una posición social mejor que la actual"), con la superación de problemas personales. También son de destacar las altas correlaciones entre los cuatro factores.

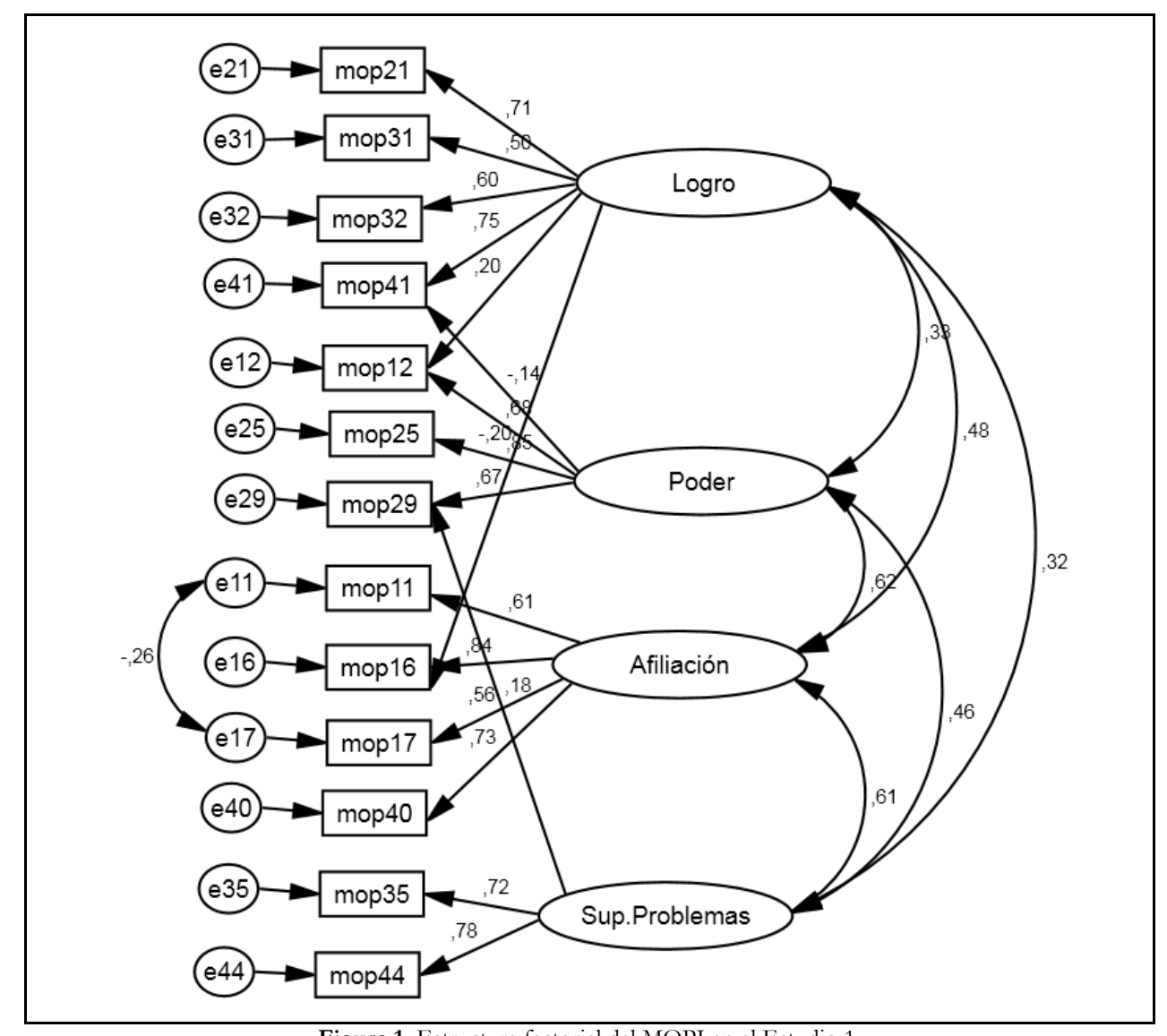

Figura 1. Estructura factorial del MOPI en el Estudio 1. 


\section{Análisis de estabilidad y comparación entre hombres y mujeres}

Con la media de estos ítems que resultaron del $A F C$ calculamos una puntuación para cada factor y realizamos un análisis con una submuestra de 73 alumnos del curso 20092010, a la que se le administró el MOPI seis meses después de la primera ocasión. El objetivo era conocer la estabilidad de la medida de dichas metas, esto es, si transcurrido un periodo suficientemente grande para conocer qué es lo que les ofrecía su primer curso en la universidad, los estudiantes seguían deseando satisfacer las mismas metas. En la Tabla 4 aparecen los valores de las correlaciones antes y después, así como los valores de las $\alpha$ de Cronbach para cada uno de los factores en la primera y segunda pasada.

Como se puede observar en la Tabla 4 , los valores de las correlaciones se sitúan entre .60 y .66 que son bastante altas después de transcurrido un periodo de seis meses. Los valores de las $\alpha$ de Cronbach también nos indican que la medida se mantiene estable a lo largo del primer curso, a excepción del factor de logro, que tiene valores inferiores a .70.

Tabla 4. Correlaciones test-retest (noviembre-mayo) y $\alpha$ de Cronbach de los factores del Cuestionario MOPI de una submuestra de 73 alumnos del Estudio 1.

\begin{tabular}{lcccc}
\hline & $\begin{array}{c}\text { Factor I } \\
\text { Logro }\end{array}$ & $\begin{array}{c}\text { Factor II } \\
\text { Poder }\end{array}$ & $\begin{array}{c}\text { Factor III } \\
\text { Afiliación }\end{array}$ & $\begin{array}{c}\text { Factor IV } \\
\text { Superar } \\
\text { Problemas }\end{array}$ \\
\cline { 2 - 5 }$\alpha$ Antes & .60 & .80 & .70 & .70 \\
$\alpha$ Después & .62 & .75 & .66 & .86 \\
Correlación Antes- & $.61^{* *}$ & $.66^{* *}$ & $.62^{* *}$ & $.60^{* *}$ \\
Después & & & & \\
\hline$* p<.01$ & & & & \\
& &
\end{tabular}

Para la comparación entre hombres y mujeres seleccionamos aleatoriamente un grupo de 134 mujeres (aproximadamente el número de hombres presentes en la muestra), distribuidas según su presencia en las submuestras de cada año. Con estos dos grupos (la totalidad de los hombres y la selección de las mujeres) realizamos una comparación de las puntuaciones en los cuatro factores finales. En ninguno de ellos encontramos diferencias significativas $(\mathrm{F}>.10)$.

\section{Estudio 2}

En este segundo estudio, los alumnos del primer curso del grado de Psicología, cumplimentaron, además del MOPI, otras escalas para determinar sus metas vitales, su percepción sobre la satisfacción de las necesidades psicológicas de autonomía, competencia y vinculación, sus tendencias conductuales de aproximación $v$ s. evitación, su nivel de perfeccionismo y su evaluación de su bienestar emocional.

Tal como adelantamos en la introducción, existen numerosos estudios que avalan la relación entre metas intrínsecas, satisfacción de las necesidades psicológicas y bienestar emocional (Deci y Ryan, 2000; Ryan y Deci, 2009; Vansteenkiste, Lens y Deci, 2006). Nuestras predicciones eran que existirían relaciones positivas entre los factores de logro, afiliación y poder (en los que se agrupan las metas sociales) con las metas vitales, la satisfacción de las necesidades, las tendencias aproximativas y el bienestar emocional. En cambio, deberían aparecer relaciones negativas entre el factor 3 (de superación de problemas personales), la satisfacción de necesidades psicológicas, los aspectos aproximativos, el bienestar y el nivel de perfeccionismo. La incorporación de esta última variable supone cierta novedad en la investigación actual y merece una explicación más detallada.

El perfeccionismo se define como una tendencia disposicional a la búsqueda de la perfección, la intolerancia a los errores y a una evaluación de sí mismo excesivamente crítica (Hewit y Flett, 1991). Tradicionalmente se ha estudiado como una característica de la personalidad asociada a trastornos psicológicos y con consecuencias negativas como ansiedad o agresividad. Sin embargo, recientemente algunos autores defienden que se puede distinguir aspectos positivos y negativos del perfeccionismo. Por ejemplo, la búsqueda de estándares altos de excelencia no implica siempre una evaluación crítica del self, y puede correlacionar positivamente con el ajuste personal (Stoeber y Otto, 2006). En cambio, el perfeccionismo que proviene de la presión externa a conseguir unos niveles altos de ejecución sí está relacionado con autoevaluaciones muy exigentes y con emociones negativas (Stoeber y Otto, 2006).

Desde esta perspectiva se han estudiado las relaciones entre las diferentes dimensiones del perfeccionismo y el ajuste académico (Shim y Fletcher, 2012, Stoeber y Stoeber, 2009). En particular, el trabajo de Shim y Fletcher (2012) mostró que la búsqueda de estándares se podía predecir a partir de metas sociales deseables como búsqueda de intimidad, desarrollo social o cuidar de los otros. En terminología de la TAD, metas intrínsecas. En cambio, la dimensión de preocupación por los errores se relacionaba con metas de dominancia, popularidad, liderazgo, búsqueda de juicios positivos y evitación de valoraciones negativas, metas, todas ellas de naturaleza extrínseca, con un excesivo énfasis en el éxito social.

Tomando como referencia estos trabajos, esperábamos encontrar relaciones positivas entre: (a) La dimensión de búsqueda de estándares de excelencia y los factores de logro; y (b) El factor de superación de problemas personales y el factor de poder (por un lado) y las dimensiones más negativas del perfeccionismo, como son la preocupación por los errores y la búsqueda de perfección, por otro.

\section{Método}

\section{Participantes}

140 estudiantes de primero de grado de Psicología de la universidad de La Laguna, del curso 2010-11. Del total de participantes el $71.4 \%$ eran mujeres. La edad media fue de 19.3 años $(D T=2.8)$. Todos participaron de forma voluntaria como forma de obtener créditos extras en una asignatura. 


\section{Instrumentos y Procedimiento}

\section{La escala MOPI.}

2. Cuestionario de Nivel de Aspiración para evaluar las Metas Vitales de los estudiantes (Grouzet, et al., 2005). Se utilizó para conocer las metas de los estudiantes en once dominios. Se les preguntaba por la importancia y la probabilidad de elegir un conjunto de 57 metas futuras. Por ejemplo "Que siempre exista alguien cerca de mi para cuidarme" o "Ser eficiente". Los autores agrupan las metas en cuatro cuadrantes que resultan de cruzar la dimensión intrínseco-extrínseco y la dimensión yo-trascendente y yo-físico. Para simplificar nuestros análisis, tal como aparece en la Tabla 5, agrupamos las metas en intrínsecas (comunidad, afiliación, autoaceptación, seguridad y salud física), extrínsecas (conformidad, popularidad, imagen y éxito financiero), espiritualidad y hedonismo. Esta escala se validó en el estudio de Grouzet et al., realizado en quince países entre los que se encontraba España. Los índices de fiabilidad de esta escala y de todas las utilizadas en este estudio también figuran en la Tabla 5.

3. Escala de Necesidades Psicológicas Básicas (BANES: Basic Need Satisfaction in General; Deci y Ryan, 2000). Mide el grado en que se experimenta la satisfacción de cada una de las necesidades psicológicas organísmicas definidas en la TAD. Es decir, en qué medida las personas sienten satisfechas las tres necesidades básicas de autonomía, competencia y vinculación. Los participantes tienen que valorar en una escala Likert del 1 al 7 frases del tipo "Me siento libre de decidir cómo quiero vivir mi vida" o "Me siento presionado".

4. Escala para valorar la tendencia conductual hacia la Aproximación o la Evitación (BIS/BAS Scale; Carver y White, 1994). Mide la sensibilidad de los sistemas de aproximación/evitación. Se trata de comprobar las diferencias individuales en el sistema BAS (que guía la actuación para conseguir lo que se desea) y en el BIS, destinado a alejarse de las situaciones indeseables. Ejemplos de ítems son: "Me desvío de mi camino por conseguir lo que quiero" o "Me duelen mucho las críticas o que me regañen". Se pide a los sujetos que evalúen su acuerdo con estas frases en una escala tipo Likert del 1 al 4.

5. Escala para medir el bienestar subjetivo (SPANAS; Joiner, Sandín, Chorot, Lostao y Marquina, 1997). Utilizamos la adaptación al español de la escala PANAS diseñada para evaluar las emociones positivas y negativas que sienten las personas en su vida cotidiana. La escala incluye 10 ítems tipo Likert para evaluar las emociones positivas, PA (en qué medida una persona se siente activa, entusiasta y alerta) y 10 ítems para evaluar las emociones negativas, NA (en qué medida la persona se siente culpable, enfadada o triste).

6. Escala de Felicidad de Fordyce (1988). Consta de una única pregunta: "En general ¿cómo te sientes normalmente de feliz/infeliz? Las respuestas van de 0 (extremadamente infeliz) a 10 (extremadamente feliz).
Tabla 5. Medias, desviaciones, asimetría, curtosis e índices de fiabilidad de cada una de las variables utilizadas en el Estudio 2.

\begin{tabular}{|c|c|c|c|}
\hline Escalas & Media & DT & $\alpha$ Cronbach \\
\hline \multicolumn{4}{|l|}{$\overline{M O P I}$} \\
\hline Metas Logro & 5.55 & .92 & .72 \\
\hline Metas SuperarProblema & 2.95 & 1.50 & .81 \\
\hline Metas Afiliación & 4.00 & 1.35 & .68 \\
\hline Metas Poder & 4.18 & 1.64 & .84 \\
\hline \multicolumn{4}{|l|}{ Nivel de aspiración } \\
\hline Metas ExtrínsecasImp & 5.48 & 1.45 & .87 \\
\hline Metas ExtrínsecasProb & 4.76 & 1.06 & .79 \\
\hline Metas IntrínsecasImp & 7.67 & .76 & .73 \\
\hline Metas IntrínsecasProb & 6.17 & .85 & .76 \\
\hline Metas EspirtualidImp & 3.09 & 1.77 & .86 \\
\hline Metas EspiritualidProb & 2.55 & 1.54 & .85 \\
\hline Metas HedonismoImp & 6.93 & 1.31 & .75 \\
\hline Metas HedonismoProb & 6.03 & 1.32 & .77 \\
\hline \multicolumn{4}{|l|}{ Necesidades psicológicas } \\
\hline Nec. Autonomía & 4.91 & .92 & .68 \\
\hline Nec. Competencia & 4.76 & .84 & .69 \\
\hline Nec.Vinculación & 5.37 & .73 & .71 \\
\hline \multicolumn{4}{|l|}{ Aproximación / Evitación } \\
\hline BASdrive & 2.68 & .52 & .53 \\
\hline BASfun & 2.90 & .53 & .51 \\
\hline BASrecompensa & 3.37 & .43 & .59 \\
\hline BIS & 3.02 & .54 & .74 \\
\hline \multicolumn{4}{|l|}{ Bienestar Subjetivo } \\
\hline Emociones Positivas & 4.42 & .66 & .72 \\
\hline Emociones Negativas & 3.30 & .95 & .85 \\
\hline Felicidad & 6.86 & 1.57 & \\
\hline \multicolumn{4}{|l|}{ Perfeccionismo } \\
\hline Estándar Personal & 2.80 & .60 & .76 \\
\hline Búsqueda Perfección & 1.98 & .59 & .81 \\
\hline Preocupa Errores & 2.04 & .68 & .82 \\
\hline
\end{tabular}

7. Escala multidimensional sobre pensamientos perfeccionistas (MPCI-E; Stoeber, Kobori y Tanno, 2010). Se trata de una escala desarrollada en Japón y traducida posteriormente al inglés. Mide la frecuencia de pensamientos asociados a un perfeccionismo disposicional en tres dimensiones diferenciadas: estándares personales, búsqueda de la perfección y preocupación por los errores. Cada una de las de las dimensiones incluye 5 ítems tipo Likert donde cada persona valora en una escala de 1-4 en qué medida se identifica con 15 frases del tipo: "Conseguir las notas más altas es para mi propio beneficio" para la primera dimensión que busca evaluar los pensamientos relacionados con los estándares perfeccionistas. La segunda dimensión evalúa los pensamientos relacionados con la necesidad de ser perfectos/as, uno de los ítems es "Debo ser perfecto/a a cualquier precio". Por último, el ítem "Si no puedo hacer algo bien es que estoy por debajo de la media" es un ejemplo de los pensamientos asociados a la tercera dimensión, relacionada con los pensamientos y emociones negativas asociadas a cometer errores. 


\section{Resultados}

\section{Análisis confirmatorio del MOPI}

Con el fin de evaluar la estabilidad de los factores encontrados en el análisis factorial del Estudio 1, realizamos un nuevo $A F C$ con la muestra del Estudio 2. En este caso el coeficiente Mardia fue inferior a 5 (c.r $=4.08)$ por lo que uti- lizamos el método de estimación de máxima verosimilitud. Igual que ocurrió en el Estudio 1 pusimos a prueba más de un modelo. El tercer modelo puesto a prueba que se expone en la Figura 2 consiguió los valores adecuados de ajuste, $\chi^{2} /$ g.l. $=116.445 / 84=1.39, C F I=0.95, I F I=0.95, G F I=0.90 \mathrm{y}$ RMSE $A=0.052$.

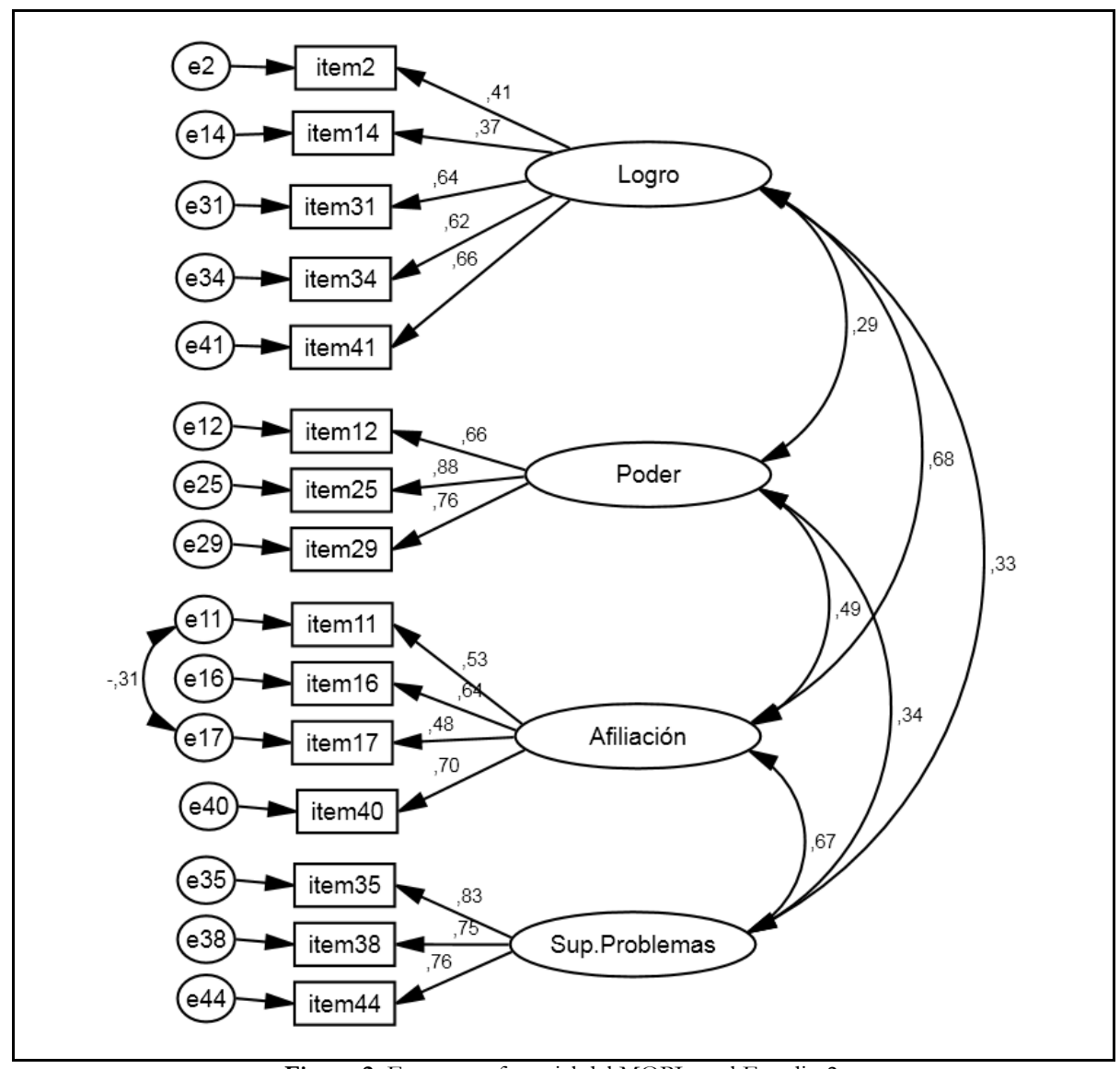

Figura 2. Estructura factorial del MOPI en el Estudio 2.

Las diferencias encontradas con respecto al $A F C$ del Estudio 1 se centran en el factor 1 de logro, que ahora incluye los ítems 2 y 14 y excluyen al 32, y el factor de superación de problemas, que incluye el ítem 38. También se eliminan las cargas cruzadas de los ítems 29 y 16. Con la media de estos ítems que resultaron del $A F C$ calculamos una puntuación para cada factor que utilizamos en los análisis de aquí en adelante.

Análisis de los factores del MOPI y el resto de las variables utilizadas

En la Tabla 5 aparecen las medias, desviaciones e índices de frabilidad de cada una de las medidas utilizadas en este estudio, y en la Tabla 6 las correlaciones significativas de cada factor del MOPI, esto es, de cada grupo de metas sociales- interpersonales con el resto de las variables utilizadas. La correlación más alta .56 , aparece entre las metas de poder y la importancia concedida a las metas extrínsecas. Con valores $\geq .30$ encontramos: la misma relación señalada con anterioridad pero ahora con la medida de la probabilidad de conseguirlo. Es decir, los estudiantes que tienen metas de poder también conceden gran importancia a las metas extrínsecas y piensan que las pueden conseguir. En el caso de las metas de logro, estos valores de las correlaciones aparecen para la importancia dada a las metas intrínsecas $\mathrm{y}$, tal como esperábamos, con la dimensión del perfeccionismo de búsqueda de estándares de excelencia. Por último, las metas de superación de problemas personales presentan correlaciones negativas con la satisfacción de las necesidades de autonomía y vinculación así como con la escala de felicidad. 
Para terminar realizamos una serie de análisis de regresiones múltiples tomando como variables dependientes las metas sociales-interpersonales y como independientes las metas vitales, la satisfacción de las necesidades psicológicas y las dimensiones del perfeccionismo. Como se muestra en la Tabla 7, las metas de Poder se pueden predecir en un $36 \%$ por la presencia de metas extrínsecas e intrínsecas. En el caso de las metas de Logro, además de la presencia de metas intrínsecas y espirituales es necesario considerar la dimensión de búsqueda de estándares, que también aparece como predictor de las metas de Superación de Problemas personales junto a la insatisfacción en las necesidades de autonomía y vinculación y la presencia de metas espirituales.

Tabla 6. Correlaciones significativas de los cuatro factores del Cuestionario MOPI con el resto de las variables del Estudio 2.

\begin{tabular}{|c|c|c|c|c|}
\hline & Logro & Poder & Afiliación & SuperarProb \\
\hline Metas ExtrínsecasImp & $.23 * *$ & $.56^{* *}$ & & \\
\hline Metas ExtrínsecasProb & & $.30^{* *}$ & & \\
\hline Metas IntrínsecasImp & $.32 * *$ & & & $-.18 *$ \\
\hline Metas IntrínsecasProb & $.19 *$ & & & \\
\hline Metas EspirtualidImp & $.21 *$ & & $.21^{*}$ & $.20^{*}$ \\
\hline Metas EspiritualidProb & $.22 * *$ & & $.18^{*}$ & \\
\hline Metas HedonismoImp & & $.24 *$ & & $-.19 *$ \\
\hline Metas HedonismoProb & & & & \\
\hline Nec. Autonomía & & & & $-.32 * *$ \\
\hline Nec. Competencia & $.19 *$ & & & $-.25^{* *}$ \\
\hline Nec.Vinculación & & & & $-.32^{* *}$ \\
\hline BASdrive & $.24 * *$ & $.23^{*}$ & & \\
\hline BASRecompensa & $.26 * *$ & & & \\
\hline Emociones Negativas & & & & $.22^{* *}$ \\
\hline Felicidad & & $-.17^{*}$ & & $-.34 * *$ \\
\hline Estándar Personal & $.33 * *$ & $.27 * *$ & $.17^{*}$ & $.22 * *$ \\
\hline Búsqueda Perfección & & & & $.22 * *$ \\
\hline Preocupa Errores & & & & $.26 * *$ \\
\hline
\end{tabular}

${ }^{* *} p<.01,{ }^{*} p<.05$

Tabla 7. Análisis de regresiones múltiples de los factores del Cuestionario $M O P I$, en función de las metas vitales, las necesidades psicológicas y las dimensiones del perfeccionismo.

\begin{tabular}{lllll}
\hline Variable & $\mathrm{B}$ & ET B & $\mathrm{B}$ & $\mathrm{R}^{2}$ \\
\hline Metas Poder & & & & $.36^{* *}$ \\
MetasExtrinsI & .90 & .12 & $.80^{* *}$ & \\
MetasIntrinsI & -.66 & .20 & $-.31^{* *}$ & \\
MetasIntrinsP & .48 & .18 & $.25^{*}$ & \\
MetasExtrinsP & -.34 & .16 & $-.21^{*}$ &
\end{tabular}

\section{Metas Logroa}

MetasIntrins

PersonalStand

$.34 \quad .09 \quad .28 * *$

MetaEspirtual

$.42 \quad .11$

$.28 * *$

$.09 \quad .04 \quad .18^{*}$

\section{Metas SuperarProblb}

\begin{tabular}{lccc} 
Autonomía & -.42 & .15 & $-.24^{* *}$ \\
Vinculación & -.40 & .20 & $-.18^{*}$ \\
MetasEspiritu & .15 & .07 & $.17^{*}$ \\
PersonalStand & .53 & .20 & $.20^{* *}$ \\
\hline
\end{tabular}

${ }^{a} F_{(6,139)}=12.63,{ }^{b} F_{(6,139)}=9.14,{ }^{c} F_{(6,139)}=20.13$

${ }^{*} p<.05, * * p<.001$

\section{Discusión}

El trabajo que presentamos aquí pretende contribuir al conocimiento de las metas de los estudiantes de psicología y cómo estas pueden influir en su vida académica. Básicamente podemos decir que cuando entran en la universidad los estudiantes agrupan sus metas en cuatro categorías claras:

(a) Metas de logro, orientadas al mundo académico, la búsqueda de conocimientos y la demostración de sus capacidades.

(b) Metas de poder, relacionadas con el liderazgo y el prestigio social y económico.

(c) Metas de afiliación y vinculatorias, con las que se busca hacer nuevas amistades y tener relaciones con una buena comunicación.

(d) Metas relacionadas con la superación de problemas emocionales e interpersonales, o preocupaciones que los estudiantes piensan que podrán resolver al estudiar psicología.

Los análisis factoriales realizados y el test-retest con una submuestra de los participantes nos informan de la estabilidad de estas metas a lo largo de todo el primer curso en la universidad.

A diferencia del trabajo inicial de los autores del MOPI (Gámez y Marrero, 2000) no hemos encontrado diferencias de género en la importancia dada a los diferentes contenidos de las metas. Esto hay que tomarlo con precaución pues sabemos que hay datos contradictorios al respecto. Recientemente Alonso-Tapia, Huertas y Ruiz (2010) encontraron diferencias significativas entre estudiantes universitarios cuando se trata de predecir el logro académico a partir del "deseo de tener éxito en la esfera pública". Esta meta de rendimiento aproximativa sólo tenía efectos beneficiosos para "los" estudiantes y no cuando se tenían en cuenta a "las" estudiantes. Sin embargo uno de los autores, en otro trabajo sobre las situaciones de interacción y las metas sociales en la adolescencia, no informó de ninguna diferencia de género a pesar de contar con una muestra equiparada en esta variable (Alonso-Tapia y Rodríguez-Rey, 2012).

La primacía del factor de Logro frente al resto de los factores encontrado no es de extrañar cuando consideramos la importancia de estas metas en los estudiantes (Elliot, 1999; Valle et al., 2009). En el caso de las metas de logro, como era de esperar, se confirma su relación con la satisfacción de la necesidad de competencia, con los aspectos aproximativos y de búsqueda de recompensa y, tal como comentamos en la introducción al Estudio 2, con la dimensión de estándar personal del perfeccionismo (Stoeber y Otto, 2006). Tampoco nos extraña que correlacione con la presencia de metas extrínsecas como el dinero o tener una imagen popular entre los compañeros. Sin embargo, lo que nos resulta más llamativo, a la luz de los análisis de regresión, es que este factor está también bastante relacionado con las metas intrínsecas. Esto significaría que el Logro al que aspiran los estudiantes también se relaciona con el crecimiento personal, la partici- 
pación en la comunidad o la búsqueda de relaciones interpersonales. También explicaría por qué hay algunos ítems que saturan en este factor y se relacionan con otros logros que no son estrictamente académicos sino que tienen una naturaleza afiliativa. Es decir, para los estudiantes de psicología, al igual que para el resto de las personas, querer ayudar a los demás o ser solidarios son metas claramente vinculatorias. La novedad es que también se entienden como logros interpersonales, logros que te pueden hacer llegar a los criterios de excelencia que marca el grupo del que quieres formar parte. También es de destacar la correlación de las metas de logro con las metas espirituales. Esto es, los estudiantes aspiran a que sus actos tengan relación con sus creencias espirituales o religiosas.

En el caso de las metas de Poder encontramos una relación significativa con la importancia concedida a las metas extrínsecas y hedonistas. Los estudiantes que se orientan hacia estas metas buscan el dinero, el prestigio y pasárselo bien. A partir de los resultados en los análisis de correlación y de regresión se diría que tener metas de poder es incompatible con darle importancia a las metas intrínsecas, puesto que, aparentemente, se sobrevaloran la importancia y la probabilidad de conseguir este tipo de metas. Shim y Flether (2012) encontraron que la dimensión de estándar personal del perfeccionismo se relacionaba positivamente con la meta social de liderazgo y negativamente con la meta de dominancia. Nuestros resultados indican una relación positiva entre Poder y estándar de excelencia aunque en nuestro caso ambas metas estaban incluidas en la dimensión de Poder. Otro dato a tener en cuenta con este factor es que tener estas metas está relacionado con una baja percepción de felicidad.

Por último, el cuarto factor, las metas de Superación de Problemas afectivos, probablemente la novedad más singular de este estudio, se relaciona con muchas variables de forma negativa. Los estudiantes con estas metas no le dan importancia ni a las metas intrínsecas ni a las hedonistas: sólo parecen mantener las espirituales. Ello probablemente esté relacionado con su vivencia subjetiva emocionalmente conflictiva mediante la cual no creen que puedan aspirar a formar parte de la comunidad o a pasárselo bien. Por supuesto, se sienten infelices, con emociones negativas y sin satisfacer ninguna de las necesidades psicológicas de autonomía, vinculación y competencia. Relacionado con este perfil de negatividad, la presencia de estas metas se relaciona con las tres dimensiones del perfeccionismo, están preocupados por los errores y al mismo tiempo buscan el perfeccionismo y la excelencia. Con estos resultados no es difícil imaginar que aquellos estudiantes que buscan resolver sus problemas afectivos e interpersonales estudiando esta carrera van a tener más interferencias que aquellos cuyas prioridades estén en las metas de Logro o Poder.

En el trabajo original de Gámez y Marrero (2000) los autores proponían que esta dimensión de Superación de pro- blemas podría estar relacionada con una mayor sensibilidad de los estudiantes de psicología hacia los temas afectivos, desestimando los aspectos negativos de esta dimensión. En este momento y atendiendo a los resultados del Estudio 2 tenemos que tener en cuenta estos aspectos negativos y preguntarnos de dónde provienen. Castro y Sánchez (2000) encontraron que tanto la salud física como la psicológica constituían dos de los objetivos vitales más importantes para los estudiantes universitarios. Es más, estos objetivos estaban relacionados con otros objetivos futuros, de tal forma que, a mayor autopercepción de salud psicológica parece que se ha logrado más en la vida y se espera lograr también más a largo plazo. También sabemos por la TAD que la satisfacción de las necesidades de autonomía, competencia y vinculación es determinante para un aprendizaje significativo y exitoso (León, Domínguez, Núñez, Pérez y Martín-albo, 2011; Niemec y Ryan 2011). Por último, hemos conocido por el estudio de Pereda, Forns y Abad (2013), con una muestra de 1033 estudiantes universitarios españoles, que el 73\% había experimentado entre uno y cinco acontecimientos potencialmente traumáticos, del tipo muerte de seres queridos, enfermedades, agresiones, accidentes de coche o agresiones sexuales. Aunque la propia definición de estos acontecimientos es polémica, no sería descabellado pensar que estos acontecimientos han podido contribuir a la importancia concedida a estas metas interpersonales que aparecen en el MOPI.

Una limitación clara de este trabajo es que la escala MOPI sólo pregunta por la importancia de las metas, por cómo los estudiantes se identifican con cada una de las metas que expresa cada uno de los ítems. Tal como hemos comprobado con el cuestionario para evaluar las metas vitales de Grouzet, et al. (2005) es interesante valorar de forma independiente la importancia y la probabilidad de conseguir una meta. En esta misma línea sería interesante indagar sobre la percepción de los estudiantes de los agentes facilitadores y obstaculizadores de dichas metas, así como de la satisfacción de aquellas conseguidas una vez finalizado el primer año en la universidad.

Por último, una pregunta que nos asalta como profesores que impartimos asignaturas en el primer año del grado de psicología es para qué podría servir los resultados de este trabajo. Creemos que conocer con más detalle el perfil motivacional de los estudiantes en su primer año en la universidad puede ayudar a diseñar los planes de orientación y acción tutorial que se están implantado en las universidades españolas. Se podría asesorar a los estudiantes en su primer curso sobre sus metas, motivos y aspiraciones iniciales y cómo se conectan con su rendimiento y bienestar. Esto no sólo podría representar un indicador de calidad de los diferentes centros, sino que se puede entender también como un instrumento pedagógico que contribuya a motivar e implicar a los alumnos en su formación académica. 


\section{Referencias}

Alonso-Tapia, J. A., Huertas, J. A y Ruiz, M. A. (2010). On the nature of motivational orientations: implications of assessed goals and gender differences for motivational goal theory, The Spanish Journal of Psychology, 13, 232-243. Doi:10.1017/S1138741600003814

Alonso-Tapia, J., y Rodriguez-Rey, R. (2012). Situaciones de interacción y metas sociales en la adolescencia: desarrollo y validación inicial del cuestionario de metas sociales (CMS). Estudios de Psicología, 33, 191-206. Doi:10.1174/021093912800676484

Bentler, P. M. (1990). Comparative fit indexes in structural models. Psychological Bulletin, 107, 238-246. Doi:10.1037/003329091072238

Byrne, B. M. (2010). Structural equation modeling with AMOS, $2^{\mathrm{a}}$ Ed. Nueva York: Taylor \& Francis.

Carver, C. S., y White, T. L. (1994). Behavioral inhibition, behavioral activation, and affective responses to impending reward and punishment: The BIS/BAS scales. Journal of Personality and Social Psychology, 67, 319-333. Doi:10.1037/00223514672319

Castro, A. y Sánchez, M. P. (2000). Objetivos de vida y satisfacción autopercibida en estudiantes universitarios. Psicothema, 12, 87-92.

Covington, M. V. (2000). Goal theory, motivation, and school achievement: An integrative review. Annual Review of Psychology, 51, 171-190. Doi: 10.1146/annurev.psych.51.1.171

Deci, E. L., y Ryan, R. M. (2000). The "what" and the "why" of goal pursuits: Human needs and the self-determination of behavior. Psychological Inquiry, 11, 227-268. Doi: 10.1207/S15327965PLI1104_01

Elliot, A. (1999). Approach and avoidance motivation and achievement goals. Educational Psychologist, 34, 169-189. Doi: 10.1207/s15326985ep3403_3

Elliot, A. J., y McGregor, H. A. (2001). A 2x2 achievement goal framework. Journal of Personality and Social Psychology, 80, 501-519. Doi: $10.1037 / 00223514803501$

Elliot, A.J., y Moller, A. (2003). Perfomance-approach goals: good or bad forms of regulation? International Journal of Educational Research, 39, 339-356. Doi: 10.1016/iijer200406003

Fordyce, M. (1988). A review of research on the happiness measures: a sixty second index of happiness and mental health. Social Indicators Research, 20, 355381. Doi: $10.1007 /$ BF00302333

Gámez, E y Marrero, H. (2000). Metas y motivos en la elección de la carrera de psicología. Revista Electrónica de Motivación y Emoción, R.E.M.E, 3, Recuperado de http://reme.uji.es

Gámez, E., y Marrero, H. (2003). Metas y motivos en la elección de la carrera universitaria: un estudio comparativo entre Psicología, Derecho y Biología. Anales de Psicología, 19,121-131.

Grouzet, F. M. E., Kasser, T., Ahuvia, A., Fernandez-Dols, J. M., Kim, Y., Lau, S., Ryan, R. M., Saunders, S., Schmuck, P., y Sheldon, K. (2005). The structure of goal contents across 15 cultures. Journal of Personality and Social Psychology, 89, 800-816. Doi: 10.1037/00223514895800

Hewitt, P. L., y Flett, G. L. (1991). Perfectionism in the self and social contexts: conception, assessment, and association with psychopathology. Journal of Personality and Social Psychology, 60, 456-470. Doi: 10.1037/00223514603456

$\mathrm{Hu}, \mathrm{L}-\mathrm{T}$., y Bentler, P. M. (1999). Cutoff criteria for fit indexes in covariance structure analysis: conventional criteria versus new alternatives. Structural Equation Modeling, 6, 1-55. Doi: 10.1080/10705519909540118

Joiner, T. E., Sandín, B., Chorot, P., Lostao, L., y Marquina, G. (1997). Development and factor analytic validation of the SPANAS among women in Spain: (More) cross-cultural convergence in the structure of mood. Journal of Personality Assessment, 68, 600-615. Doi: 10.1207/s15327752jpa6803_8

Jöreskog, K. G., y Sörbom, D. (1993). LISREL 8: Structural equation modeling with the SIMPLIS command language: Chicago: Scientific Software International.

Kasser, T., y Ryan, R. M. (1996). Further examining the American dream: Differential correlates of intrinsic and extrinsic goals. Personality and Social Psychology Bulletin, 22, 280-287. Doi: : 10.1177/0146167296223006

León, J., Domínguez, E., Núñez, J. L., Pérez, A., y Martín-Albo, J. (2011). Traducción y validación de la versión española de la Echelle de Satisfaction des Besoins Psychologiques en el contexto educativo, Anales de Psicología, 27, 405-411.

Linnenbrink, E. A., y Pintrich, P. R. (2002). Motivation as an enabler for academic success. School Psychology Review, 31, 313-327.
Midgley, C. (Ed.). (2002). Goals, goal structures and patterns of adaptative learning. Mahwah, NJ: Lawrence Erlbaun Associates.

Moscowitz, G. B., y Grant, H.(Eds). (2009). The psychology of goals. Nueva York: Guilford.

Niemec, C. P., y Ryan, R. M. (2009). Autonomy, competence and relatedness in classroom. Theory and Research in Education, 7, 133-144. Doi: $10.1177 / 1477878509104318$

Nurmi, J-E. (1992). Age differences in adults' life goals, concerns, and their temporal extension: A life course approach to future-oriented motivation. International Journal of Behavioral Development, 15, 487-508. Doi: $10.1177 / 016502549201500404$

OECD (2011). Education at a Glance 2011: OECD Indicators, OECD Publishing. Recuperado de http://dx.doi.org/10.1787/eag-2011-en

Pereda, N., Forns, M., y Abad, J. (2013). Prevalencia de acontecimientos potencialmente traumáticos en universitarios españoles. Anales de Psicología, 29, 178-186. Doi: http://dx.doi.org/10.6018/analesps.29.1.124921

Pintrich, P. R. (2000). Multiple goals, multiple pathways: the role of goal orientantion in learning and achievement. Journal of Educational Psycbology, 92, 544555. Doi: $10.1037 / 0022-0663.92 .3 .544$

Reeve, J. (2009). Why teachers adopt a controlling motivating style toward students and how they can become more autonomy supportive. Educational Psychologist, 44, 159-178. Doi: 10.1080/00461520903028990

Reeve, J., Nix, G., y Hamm, D. (2003). Testing models of the experience of selfdetermination in intrinsic motivation and the conundrum of choice. Journal of Educational Psychology, 95,375-392. Doi: 10.1037/0022-0663.95.2.375

Rodríguez, S., Cabanach, R. G., Piñeiro, I., Valle, A., Nuñez, J. C y GonzalezPienda, A. (2001). Metas de aproximación, metas de evitación y múltiples metas académicas. Psicothema, 13, 546-550.

Ryan, R. M., y Deci, E. L. (2009). Promoting self-determined school engagement: Motivation, learning, and well-being. En K. R. Wentzel y A. Wigfield (Eds.), Handbook on motivation at school (pp. 171-196). Nueva York: Routledge.

Shim, S. S., y Fletcher, K. L. (2012). Perfectionism and social goals: what do perfectionists want to achieve in social situations? Personality and Individual Differences, 52, 919-924. Doi: 0.1016/j.paid.2012.02.002

Shim, S. S., y Ryan, A. M. (2012). What do students want socially when they arrive at college? Implications of social achievement goals for social behaviors and adjustment during the first semester of college. Motivation and Emotion, 36, 504-515. Doi: 10.1007/s11031-011-9272-3

Stoeber, J., Kobori, O., y Tanno, Y. (2010). The Multidimensional Perfectionism cognitions Inventory-English (MPCI-E): Reliability, validity, and relationships with positive and negative affect. Journal of Personality Assessment, 92(1), 16-25. Doi: 10.1080/00223890903379159

Stoeber, J., y Otto, K. (2006). Positive conceptions of perfectionism: Approaches, evidence challenges. Personality and Social Psychology Review, 10, 295-319. Doi: $10.1207 /$ s15327957pspr1004_2

Stoeber, J., y Stoeber, F. S. (2009). Domains of perfectionism: Prevalence and relationships with perfectionism, gender, age, and satisfaction with life. Personality and Individual Differences, 46, 530-535. Doi: 10.1016/j.paid.2008.12.006

Valle, A., Cabanach, R. G., Núñez, J. C., González-Pienda, J.A., Rodríguez, S y Piñeiro, I. (2003). Multiple goals, motivation and academic learning. British Journal of Educational Psychology, 73, 71-87. Doi: 10.1348/000709903762869923

Valle, A., Núñez, J. C.,Cabanach, R. G., González-Pienda, J. A., Rodríguez, S. Rosario, P., Muñoz-Cadavid, M. A y Cerezo, R. (2009). Academic goals and learning quality in higher education students. The Spanish Journal of Psychology, 12, 96-105. Doi: 10.1017/S1138741600001517

Vansteenkiste, M., Lens, W., y Deci, E. L. (2006). Intrinsic versus Extrinsic goal contents in self-determination theory: Another look at the quality of academic motivation. Educational Psychologist, 41, 19-31. Doi: 10.1207/s15326985ep4101_4

Watkins, M. E. (2006). Determining parallel analysis criteria. Journal of modern applied statistical methods, 5, 344-346.

(Articulo recibido: 25-03-2013; revisado: 05-11-2013; aceptado: 11-03-2014) 